\title{
Optimising intensive apple crown pruning by IT improvements
}

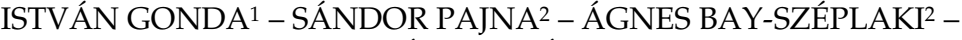 \\ MIHÁLY SZILÁGYI² \\ ${ }^{1}$ University of Debrecen, Institute of Horticulture, Debrecen, Hungary \\ 2 eKÖZIG Plc., Debrecen, Hungary \\ gonda@agr.unideb.hu
}

\begin{abstract}
Summary
Prunig is a key element of fruit production technology and is affected by a number of subjective factors. Putting research findings into practice is often hindered by bad and harmful habits that make plantations and trees heterogenious in terms of growth and fruiting features, which can even lead to significant financial losses. Having identified this challenge, in this publication we aim to present some guidelines for those who are open to new ways and believe in the power of innovation. The $21^{\text {st }}$ century is undoubtedly the century of unstoppable IT innovations filtering through into our everyday lives. The fact that our project is based on IT innovations is not only essential due to the subject matter itself but also because it makes it easier to address younger generations. Whichever tool they choose to use (smartphone, tablet, laptop), all registered students have access to our web contents, making it super easy for them to practice pruning techniques. Students don't just read texts in our training materials but have access to a pool of pictures and images to illustrate the different procecces and phenomena. All the different phrases have explanations against them, so anyone can understand the ins and outs of why and how to prune or remove the different crown parts (5-year-old and above crown parts, 24-year old parts or 1-year-old parts) to achieve specific results, whether it be apple, pear or cherry trees etc. Our database comprises thousands of pictures that will walk you through the entire fruit tree pruning process, without you even having to leave your desk. There is an old proverb that also applies to pruning: "The wise man learns from the mistakes of others, the fool has to learn from his own". Our program aims to help students by presenting a number of bad examples along with their corrections as well as photos of ideal ways to prune multiple different crown shapes. Using our web-based tool eliminates time and space limitations, i.e. you can access our interactive contents to gain or extend your pruning skills, irrespective of the pruning time and location.
\end{abstract}

Keywords: pruning, optimisation, intensive crown shapes, digital, education, innovation

\section{Introduction}

Pruning fruit trees has always been the center of attention amongst fruit producers, as constant high yielding larger and smaller crown shapes can be achieved by crown trainng and bearing treepruning. 
Compared to other aspects of production technology (weed and pest control, tree care, soil operations etc.) defining optimal pruning techniques is associated with a lot more insecurities and undecisiveness. This sector has been affected by significant labour shortage in the past few years, and backfilling well trained skilled labour is becoming more and more of an urgent matter.

Of all aspects of production technology, pruning appears to be the most subjective one, for which the simple reason is that it is the most reliant on human know-how and practice. This is the case in spite of the extensive scientific literature and physiologically accurate practical pruning guidelines that are available and widely used for the different fruit species.

Compared to other aspects of production technology, guidelines descriptions and recommendations for pruning technologies contain mainly qualitative rather than quantitative features (e.g. strong or moderate pruning, pruning back, thinning, dense, thin etc). Out of woody cultures, the only fruit types where optimal yield can be associated with quantitative indexes (bud, blossom and fruit density) to some extent are grapes and peaches.

The crown structure, size and shape of our fruit plantations show great variety even for rootstock - scioncultivar trees of the same age. The natural cause for this is partly the growing site that affects the plant's conditions (growth and fruting potential). Another factor that might cause differences is the growth and fruiting impacts of the year.

What has an even larger impact, however, is the experience, approach and pruning strategy and practices of the grower, pruner and extentionist. Different grower habits may often lead to very different plantation outlooks and results.

Experience shows that subjective i.e. human factors have a much larger impact on the heterogeneity of the trees and plantations than objective factors do. Any process, technology or solution promoting an objective approach and simple solutions/practices can significantly compensate for the lack of labour force.

\section{Subject matter}

In response to the above complex subject matter, eKÖZIG Zrt., and the Institute of Horticulture at the University of Debrecen have come up with a possible solution based on a wealth of decades of experience and knowledge base as well as the latest IT improvements.

eKözig was formed to carry out the development, implementation, operation, and work and process management of various, mainly economics and agro-informatics related systems. Our systems are 
innovation-and knowledge based and offer more efficient solutions due to a combination of IT and communication capacities and a reinterpretation of different processes. We have had great results with the IOT and artificial intelligence, and are continuously implementing our new findings in our systems.

In the focus of our project is a tree pruning training material, with special emphasis on basic know-how and a hands-on practical approach to a variety of pruning scenarios. With the help of our IT tool, teaching pruning does no longer need to be carried out on the plantation sites, which means that time and space restrictions are eliminated. Students can learn and practice pruning on any day of the year, even before the start of the pruning season. Furthermore, our project enables plantation owners to test the knowledge and skills of their workers before they start working on their plantations.

\section{Material and method}

Our project makes use of many decades' worth of knowledge and skills in the elements of the cultivation systems (crown shapes, non-bearing and bearing tree pruning methods) with special attention to those that can be applied both to present and future conditions. A large volume (several thousands) of pictures aim to help learning and understanding by presenting the morphological features of different fruits (bearing tree parts, woody parts of 1 year and above, as well as their optimal crown shapes and crown structures).

Thus, we also present crown pruning methods that are normally not even considered by professional large-scale production enterprises due to their cost implications.

Based on the above, we present optimal crown training and bearing tree pruning tricks that are applied from the beginning of plantation establishment, and also provide the opportunity to practice and test your newly aquired skills.

Our theoretical and visual materials enable our users to familiarise themselves with the below areas:

- Vegetative growth types: 5 years old and above crown parts, 2-4 year old parts, 1 year old parts;

- Fruiting tree parts and their location depending on fruit species;

- Visual appearance of vegetative-generative features, proportions of flower buds and crown parts;

- Optimal and incorrect crown shapes;

- Method and extent of correct tree pruning.

All visuals (an image pool of over 2000 and continuously growing) have short descriptions against them detailing the whys and hows. 
Further concepts with corresponding images:

1) Pre-pruning considerations,

- The visual appearance of the tree (size, shape, density etc.),

- Based on visual appearance, what pruning method to use and

- how to implement it (what steps to follow);

2) Heading back cuts or thinning out cuts;

3) Heavy pruning, moderate pruning, optimal pruning, incorrect pruning;

4) Visual appearance of the crown pre- and post-pruning.

\section{Results}

Basic principles of apple tree pruning

Our research shows that both domestic and foreign apple tree plantations are defined by the following crown shapes: free spindle, slender spindle and super spindle. These half-intensive and intensive crown shapes mainly differ in size (primarily width) but they all have their slender central leader in common. Apart from size, their differneces lie in the number (density) and length of the scaffold limbs and branches of the central leader. This build makes it necessary as well as possible to train and maintain all three crown shapes along the same principles (Figure 1, Table 1).

Figure 1. Crown shapes of the apple trees

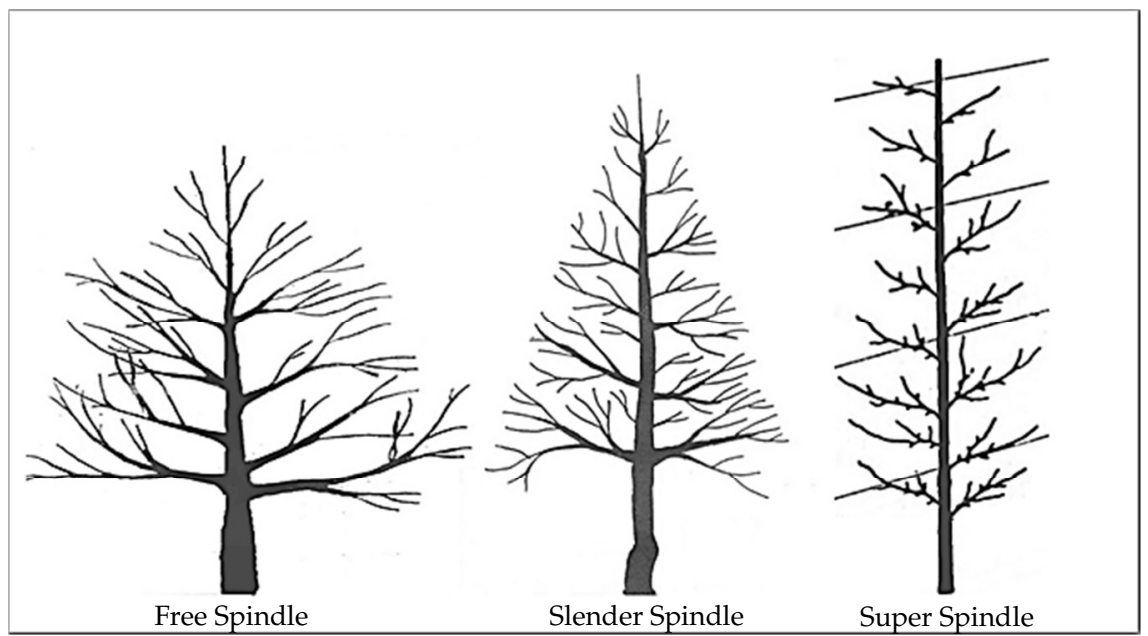


Table 1. Growth features of the different apple tree crown shapes

\begin{tabular}{|c|c|c|c|}
\hline \multirow{2}{*}{$\begin{array}{c}\text { Central leader } \\
\text { dominance }\end{array}$} & Free Spindle & Slender Spindle & Super Spindle \\
\hline & moderate & stronger & strongest \\
\hline Branch thickness & $\begin{array}{l}\text { gradually decreasing } \\
\text { from bottom to top }\end{array}$ & $\begin{array}{l}\text { bottom third is thicker, } \\
\text { rest is identical }\end{array}$ & $\begin{array}{c}\text { same thickness from } \\
\text { bottom to top }\end{array}$ \\
\hline Branch length & long & shorter & shortest \\
\hline Branch density & thin & denser & densest \\
\hline Branch life time & long & $\begin{array}{l}\text { bottom long, } \\
\text { above shorter }\end{array}$ & uniform short \\
\hline
\end{tabular}

Source: own development

A Summary of main principles:

- Selective thinning based on the thickness proportions of the central leader and branches (Zahn index).

- Consistent removal of growths of all angles that are significantly different from hortizontal (steep or downward growths).

- Thinning of all growths that are significantly different from the average branch thickness in their respective hight sector.

- Depending on crown shape, a gradual (free spindle) and moderate (slender spindle) bottom-to-top decrease of the thickness and length of the side shoots and branches.

- Creating and maintaining near identical thickness and length (cylinder shape) across the whole length of the central leader (super spindle).

- In addition to the above, fruiting branches on bearing trees need to be pruned in line with the year characteristics (high bud density) or moderately (low bud density) in order to control yield volume.

\section{Pruning perspectives for intensive crown shapes}

With the improvements in fruit production and the spread of intensive techniques, the occurrence of significantly different crown thickness and age has become lower or non-existent. Tree structure is typically characterised by a gradually ageing and thickening central leader and its 1-4 year old branches and growths that are renewed on a rotational basis.

Simpler crown shapes make it possible to maximise the implementation of pruning objectives to create and maintain homogenity for both trees and plantations.

It becomes possible to follow nearly or completely identical routine pruning steps for each tree. Simpler crown shape makes pruning simpler, easier and more efficient as well, which leads to an increase in yield as well as better fruit quality. Furthermore, pruning efficiency, i.e. the performance of the pruner is also increased. 
With the simplification of the crown shapes, trees become very clear and transparent, and just by looking at their photos, pruning tasks and optimal pruning methods can be easily defined both during crown training and in bearing tree pruning as well.

Our experience is that presenting numerous examples of pruning mistakes or - contrarily - positives will „unvoluntarily" result in better pruning solutions, as well as in developing and maintaining an optimal crown shape.

In association with the visuals, explanations, recommendations, definitions and kind-of recipes become easy to follow, which facilitates the achievement of our aim of promoting implementation by providing short but conscise task definitions.

\section{Foundations of our pruning training material}

In the planning phase of our digital pruning training material we examined the possibility of creating 3D crown shapes and prune them digitally to present various pruning tricks. However, we came to the conclusion that the positives of 3D imaging will be outweighed by its cost implications, so we decided to provide a vast pool of 2D photos instead. We believe that photos are more than sufficient to facilitate the pruning of different crown shapes.

The pruning dilemmas and mistakes shown in the photos as well as their correction is easily done via our IT tool. Furthermore, it also becomes possible to present numerous optimal shapes in the training material. Students can study a specific pruning scenario across multiple pictures and images, which guarantees that they basically can't help but will recognise them by the time they get to the end of the material and they will be able to act accordingly and take the right steps in the real-life plantation. Our objective across the entire training material is to back all descriptions up with corresponding images, which is differnt from the majority of published paper based training materials where authors have much more limited possibilities. In addition to this combination of text and associated images, another key element of our software is the pruning practise and test/exam module. Both modules are centered around "pruning" as shown and implemented in the images. Let us emphasize once again that pitcures of trees provide a perfect opportunity to present and practice pruning techniques, as principles are the same, no matter what angle we look at a tree from. We present all sorts of scenarios from unpruned tree crowns to the correction of incorrect pruning. 
In line with the tree's life cycle, we provide guidelines as to when to encourage tree growth by backpruning, when to thin crown parts and how to implement these techniques.

Students can not only study entire trees in our training material but they can also choose to investige specific tree parts and their pruning processes close-up (e.g. correct heading cuts per crown sector and age), following and practising pruning tasks from the point of plantation establishment.

Both the practice and the test/exam modules are interactive. In the practice module, all pictures have questions relating to pre-pruning considerations against them. These rather pin-pointed concrete questions aim to guide the student in the initial learning process. Later, in the test/exam module, the questions against the images are less specific, making it a bit more difficult for students to answer.

In the interactive modules students need to identify and mark the branches and other growths that they think need thinning or backpruning. It is essential that - similarly to real-life plantation operations students need to be as specific as possible when identifying the location of each cut, as the location, size and angle of a heading cut greatly affects the way the tree will react. Students can save their answers and the software will check whether the cuts were added at the right spots. If the cut locations were marked beyond a specific error range, the software will mark those as incorrect.

Students can view their correct and incorrect answers after each time they hit save, and they can view the results of optimal pruning, then they can move on to the next tree that needs pruning. When assessing the results, the software can differentiate between generic and less significant mistakes, due to weighed assessment. Thus, if a student marks a branch that absolutely needs to be removed in order to maintain the balance of the tree, they will definitely get more points than for marking the location of a cut for rejuvenation pruning. In both modules, after dozens of pictures have been marked and checked, the software adds up all the scores and shows the results. Due to the large volume of pictures and the extentability of the database, students can encounter newer and newer scenarios both in the practice and in the exam phase, which helps the knowledge and skills gained when studying each tree to really sink in.

Students almost instinctively prune tree shapes correctly after looking at as few as 20 pictures. Training students in such a consistent way can ensure that their approach to pruning will result in more homogenious plantations, thus making the long-term maintenance of plantations simpler and more manageable. 


\section{Structure of the pruning training material}

Users can access the training material from any IT device (smartphone, tablet, laptop) via an internet based web interface. After logging in, students are directed to the homepage, where they can choose from the following options:

- General information;

- Tree pruning in practice (step by step);

- Glossary of technical terms and expressions;

- Select a fruit type.

The first 3 areas above are basically text-based training materials with images and pictures to back up and explain the respective text.

The "Select a fruit type" funcionality forwards the user to a page where they can select from the fruit types included in their subscription (e.g. apple, pear, cherry, plum, etc). The student then has two options: they can either view the text based material on the relevant crown shape (e.g. free spindle, slender spindle, super spindle), or if they have already completed that material, they can opt for the general pruning practice pages that cover multiple crown shapes)

After the completion of each course material, the user is offered a test page with revision excercises to test their knowledge. Upon completion of these questions, the user's scores are shown on the results page. Based on their scores, the application either forwards the user on to the pruning practice page or they get redirected to the course material to polish their knowledge further. In the pruning practice section, students can practice pruning and take exams by pruning tree images, as described in the previous chapter. All excercise sheets have both beginner and advanced levels. After successful completion of the practice module, the user is forwarded to the exam module.

Users can easily maneuver back to the starting page from any of the pages, but they can also move straight to the course material or the exam module if needed. Similarly to the above (practice, test/exam and beginner/advanced levels), selecting the general practice functionality will prompt a comprehensive list of pruning excercises to appear, covering all crown shapes.

You can return to the training material or the practice exercises at any point, but the glossary on its own can also be a very helpful tool to use.

\section{The significance of the subject matter}

The significance of our planned project also lies in the fact that Hungary produces an annual $0.7-1$ tons of fruits on approximately 2 million production locations (private gardens, enclosed gardens, scattered 
gardens/plots, smaller or larger market gardens/plantations) These locations are extremely diverse in terms of intensity (i.e. crown size), the number of trees and planting systems. Thus, crown training and bearing tree pruning tricks and principles shown on smaller, intensive, as well as larger, extensive trees might provide useful guidance even to owners of standalone individual trees as well.

\section{Further room for improvement}

Due to the extendability of the pool of visuals, there will be a possibility for plantation owners to use photos of their own trees to train their workers before the start of the pruning season. Furthermore, our tool might enable extentionists to help plantation owners based on photos, without having to physically attend remote locations, and thus an extended version of our tool might make remote counseling possible. 